\title{
Restoration of orbicularis oculi muscle function in rabbits with peripheral facial paralysis via an implantable artificial facial nerve system
}

\author{
YAJING SUN ${ }^{1}$, CHENG JIN $^{2}$, KEYONG LI ${ }^{1}$, QUNFENG ZHANG ${ }^{3}$, LIANG GENG $^{1}$, XUNDAO LIU $^{4}$ and YI ZHANG ${ }^{2}$ \\ ${ }^{1}$ Department of Otorhinolaryngology Head and Neck Surgery, Shanghai General Hospital; \\ ${ }^{2}$ Department of Otorhinolaryngology, Shanghai Renji Hospital, Shanghai Jiao Tong University School \\ of Medicine, Shanghai 200080; ${ }^{3}$ NCC Medical Company, Shanghai 200245; ${ }^{4}$ School of Chemistry and \\ Chemical Engineering, Shanghai Jiao Tong University, Shanghai 200240, P.R. China
}

Received February 28, 2016; Accepted March 10, 2017

DOI: 10.3892/etm.2017.5223

\begin{abstract}
The purpose of the present study was to restore orbicularis oculi muscle function using the implantable artificial facial nerve system (IAFNS). The in vivo part of the IAFNS was implanted into 12 rabbits that were facially paralyzed on the right side of the face to restore the function of the orbicularis oculi muscle, which was indicated by closure of the paralyzed eye when the contralateral side was closed. Wireless communication links were established between the in vivo part (the processing chip and microelectrode) and the external part (System Controller program) of the system, which were used to set the working parameters and indicate the working state of the processing chip and microelectrode implanted in the body. A disturbance field strength test of the IAFNS processing chip was performed in a magnetic field dark room to test its electromagnetic radiation safety. Test distances investigated were 0 , 1,3 and $10 \mathrm{~m}$, and levels of radiation intensity were evaluated in the horizontal and vertical planes. Anti-interference experiments were performed to test the stability of the processing chip under the interference of electromagnetic radiation. The fully implanted IAFNS was run for $5 \mathrm{~h}$ per day for 30 consecutive days to evaluate the accuracy and precision as well as the long-term stability and effectiveness of wireless communication. The stimulus intensity (range, 0-8 $\mathrm{mA}$ ) was set every 3 days to confirm the minimum stimulation intensity which could indicate the movement of the paralyzed side was set. Effective stimulation rate was also tested by comparing
\end{abstract}

Correspondence to: Dr Keyong Li, Department of Otorhinolaryngology Head and Neck Surgery, Shanghai General Hospital, Shanghai Jiao Tong University School of Medicine, 100 Haining Road, Shanghai 200080, P.R. China

E-mail: likeyong5@126.com

Key words: peripheral facial paralysis, orbicularis oculi muscle, implanted electrodes, functional electrical stimulation, external control the number of eye-close movements on both sides. The results of the present study indicated that the IAFNS could rebuild the reflex arc, inducing the experimental rabbits to close the eye of the paralyzed side. The System Controller program was able to reflect the in vivo part of the artificial facial nerve system in real-time and adjust the working pattern, stimulation intensity and frequency, range of wave and stimulation time. No significant differences in the stimulus intensities were observed during 30 days. The artificial facial nerve system chip operation stable in the anti-interference test, and the radiation field strength of the system was in a safe range according to the national standard. The IAFNS functioned without any interference and was able to restore functionality to facially paralyzed rabbits over the course of 30 days.

\section{Introduction}

At present, there is a lack of effective clinical treatments for long-term facial nerve lesions. The predominant available therapies are facial nerve decompression surgery and facial nerve transplantation (1). However, these treatments have their own limitations. Although facial nerve decompression is a suitable therapeutic option for facial nerve damage due to trauma or other causes of facial nerve compression, it requires that the anatomy of the facial nerve must be kept intact. There is also a certain time limit (within one month) in which it should be performed, otherwise the likelihood of successful treatment is reduced (2). Facial nerve transplantation, which involves connecting the two broken ends of the facial nerve via suture, or neural transplantation (connecting the facial nerve with a nerve taken from another area of the body), is a common method used for reconstructing the facial nerve (3). However, the neural transplantation only serves as a bridge. If the break is from a superior anatomical position, it will take a long time (six months) for the facial nerve to reach the muscles (4). During this period, the motor end plate (nerve and muscle joint) may shrink, which leads to disuse atrophy. This is the main cause of permanent facial paralysis (2).

The present study explored a novel treatment strategy, which aimed to restore the facial nerve reflex arc via an 
artificial facial nerve system (AFNS). The AFNS contains a signal-collecting microelectrode, processing chip and stimulating microelectrode, as well as a system control program on a computer. The processing chip and system control program communicate with each other to process signals and control the current stimulus (5). The signal-collecting microelectrode is implanted into normal facial muscles, and receives electrical signal from normal facial muscles. This information is transported to the artificial facial nerve system processing chip. The chip analyzes this information, determines the movement patterns of the normal side, then sends a signal to the stimulating microelectrode implanted in the paralyzed muscle, maintaining a synchronous movement with the normal side.

In previous research by the current authors, the reflex arc was restored using an AFNS (5). In this previous research, the processing chip was outside of the body and was connected to a computer, in which there was a system controlling program (System Controller). This program helped us to set the working parameters and confirm the function of the AFNS.

The purpose of the present study was to implant the processing chip, together with the microelectrodes, into the paralyzed rabbits to make the system fully implantable. This was named the implantable AFNS (IAFNS). The communication system between the chip and the System Controller was changed from wired to wireless. The processing chips were improved to adapt to the wireless communication. The IAFNS was implanted into animal models in order to restore orbicularis oculi muscle function. The effectiveness, stability and security of the wireless communication between the processing chip and the System Controller was assessed in animal experiments in the current study.

\section{Materials and methods}

Animals and equipment. A total of 12 healthy male New Zealand rabbits (weight, 2-2.5 kg; age, $6 \pm 0.41$ months) were used in the present study [permission number: SCXK (SH) 2012-0007 and SYXK (SH) 2009-0086]. The temperature and humidity of the lab were $24-26^{\circ} \mathrm{C}$ and $60 \%$, respectively. Rabbit food (pellet feed as required) and water $(100 \mathrm{ml} / \mathrm{kg})$ were provided every day. The present study was approved by the Ethics Committee of Shanghai General Hospital, Shanghai Jiao Tong University School of Medicine (Shanghai, China). An SMB100A signal generator (Rohde \& Schwarz UK, Ltd., Hampshire, UK) and an E4419B EPM series power meter (Agilent Technologies, Inc., Santa Clara, CA, USA) were used for low frequency interference experiments. High frequency interference experiments were conducted using an N5181A $100 \mathrm{KHz}-6 \mathrm{GHz}$ MXG analog signal generator and an N1912A P-series power meter (Agilent Technologies, Inc. Santa Clara, CA, USA). The radiation field strength test was performed using an EMI test receiver $20 \mathrm{~Hz}-40 \mathrm{GHz}$ (ESU40; Rohde \& Schwarz UK, Ltd.).

Processing chip. The IAFNS consisted of two parts: An external part and an internal part. The external part contained the System Controller and its wireless communication chip module. The internal part contained the processing chip and microelectrodes. The communication between each part was via the wireless communication chip module. The signal from the uninjured side of the face was collected and analyzed by the processing chip. Then, a stimulating signal was sent out to stimulate the injured side (Fig. 1).

Based on previous research by the current authors (5), the processing chip consisted of three parts: The signal collection module, the stimulating module and the wired communication module. The signal collection module of the chip was composed of three microelectrodes, including two collection electrodes and one reference electrode. The stimulating module included a three-channel stimulation electrode and one reference electrode. These electrodes were made of nano platinum black, which was demonstrated to be safe and effective in previous research (6). In the current research the processing chip was implanted into the forehead of the facial nerve paralyzed animal model. The communication between the processing chip and the System Controller was changed from wired to wireless. A new communication module was added into the processing chip. Two-way data communication was established by radio frequency communication. The communication module used a CC1110 low power consumption microprocessor unit (MPU) chip (produced by Texas Instruments, Inc., Dallas, TX, USA), which contains a standard enhanced $8051 \mathrm{MCU}$ and a wireless transceiver chip CC1100, with $433 \mathrm{MHz}$ frequency-shift keying communication unit connecting with the MPU through the Serial Peripheral Interface (Fig. 2). The chip was $3 \times 2 \mathrm{~cm}$, and was implanted into the forehead of the rabbit (Fig. 3).

System Controller. The System Controller on the computer outside the body was designed to indicate the working status and control the implanted processing chip via two-way wireless data communication. This program obtained the contralateral facial excitation mode by analyzing the wavelength, voltage and frequency of the input signal, as well as the MPU output of the corresponding stimulus mode through an electrical stimulator. This program could display the normal side wavelength as well as adjust the electric detection algorithm and threshold and stimulation parameters (stimulation intensity and frequency, range of wave and stimulation time) of the implanted part, to ensure the best working state was maintained.

\section{Electromagnetism yield in radiation assessment and anti-inter-} ference tests. Electromagnetism yield in radiation assessment and anti-interference tests of the chip were performed in Shanghai Testing \& Inspection Institute for Electrical Equipment (Shanghai, China). The tests were performed in an electromagnetic darkroom $\left(26^{\circ} \mathrm{C}\right), 21.7 \times 13.0 \times 13.0 \mathrm{~m}$, produced by Albatross Projects GmbH (Nattheim, Germany). Three rabbits and three chips were used in the test.

Anti-interference test of the chip. The purpose of this test was to assess the stability of the the processing chip under the interference of electromagnetic radiation. For low anti-interference, an SMB100A signal generator (Rohde \& Schwarz UK, Ltd., Fleet, UK) and an E4419B EPM series power meter (Agilent Technologies, Inc.) were used. An N5181A 100 KHz-6 GHz MXG analog signal generator (Agilent Technologies, Inc.) and an N1912A P-series power meter (Agilent Technologies, Inc.) were employed for high frequency interference. The chip was placed on a $0.8 \mathrm{~m}$ high insulation test panel. The 


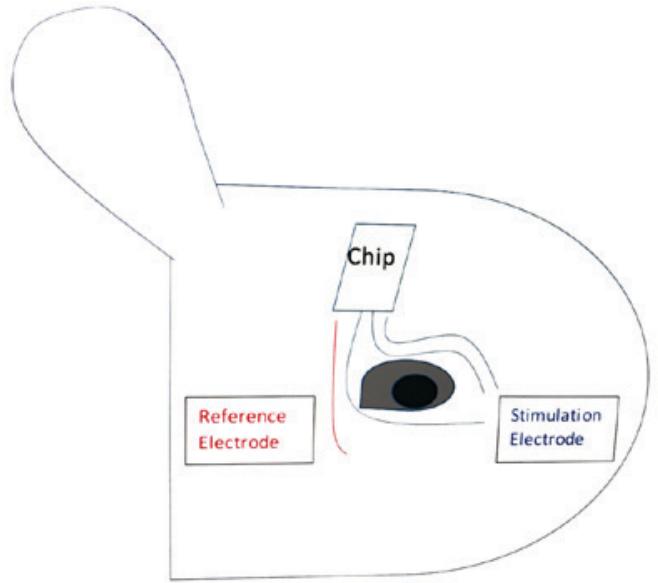

Figure 1. Diagram depicting the position of the artificial facial nerve system chip and the electrodes. The stimulation module included three-channel stimulation and one reference electrode. Two stimulating electrodes were embedded into the superior orbicularis oculi and the other into the inferior orbicularis oculi. The reference electrode was embedded into the outer canthus.

signal generator antenna was placed $3 \mathrm{~m}$ from the test chip. Pyramid-shaped absorbing material was placed between the chip and antenna. Low and high frequency tests were performed at $80 \mathrm{MHz}-1 \mathrm{GHz}$ and $1-2.5 \mathrm{GHz}$, respectively; the test field intensity was $3 \mathrm{~V} / \mathrm{m}$, the test frequency increased by $1 \%$ every time (according to the national standard YY 0505-2012). For each frequency, the scanning of amplitude modulation carrier reflected the residence time in the chip movement and response time $(\sim 0.5 \mathrm{sec})$. The test chip was assessed under two polarized states of the transmitting antenna: One antenna in the perpendicular polarization position, another in the horizontal polarization position. The anti-interference test results had four ranks: Level A, the electric device performed normally in the test; level B, the function was lost or the performance temporarily reduced, but readily restored with no need of operator intervention; level $\mathrm{C}$, the function was lost or the performance temporarily reduced, but could return to normal following operator intervention; and level D, due to hardware or software damage, or missing data, the lost function failed to return to normal.

Electromagnetism yield in radiation assessment. The electromagnetic radiation energy level produced by the chip was assessed using the ESU EMI Test Receiver $20 \mathrm{~Hz}-40 \mathrm{GHz}$ (ESU40; Rohde \& Schwarz UK, Ltd.). The chip in active status launched and received signals; every cycle had a firing time of $10 \mathrm{msec}$ and receiving time of $\sim 40 \mathrm{msec}$.

The chip functioned in three states: State 1 , in vitro environment condition (three chips); state 2 , implanted within the rabbit with the skin not sutured (three rabbits); and state 3, implanted within the rabbit with sutured skin (three rabbits). Test distances investigated were 0, 1, 3 and $10 \mathrm{~m}$ (according to the national standard YY 0505-2012).

Establishment of facial paralysis model. A total of 12 adult male New Zealand rabbits were used in facial nerve system rebuilding experiments. Following administration of $3 \mathrm{ml} / \mathrm{kg}$ $1 \%$ pentobarbital sodium via the marginal ear vein, the junction

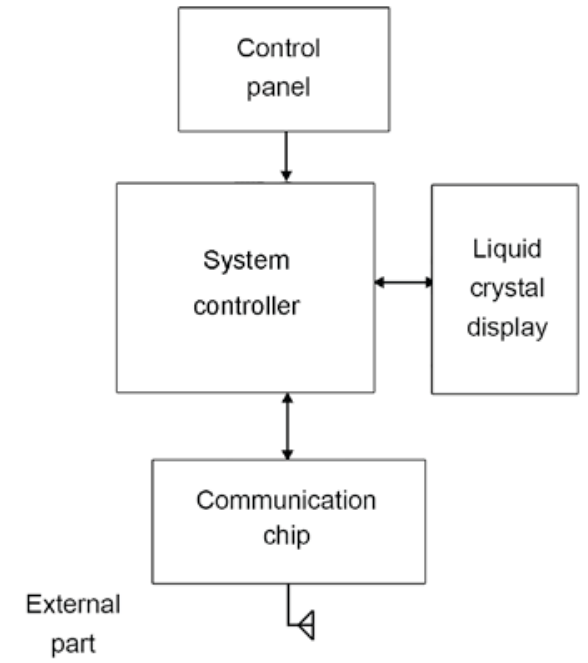

Skin

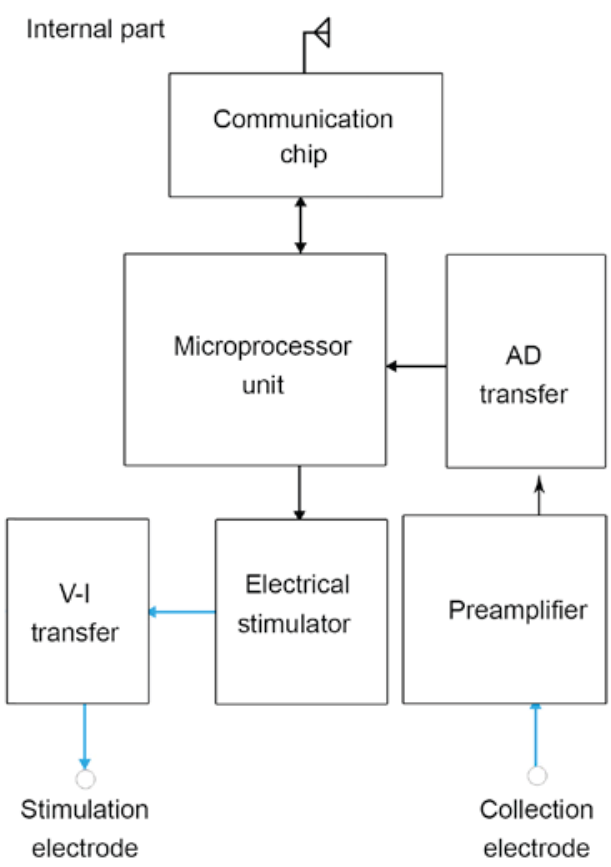

Figure 2. Flowsheet of hardware system connection. The implantable artificial facial nerve system consists of two parts: The external part and the internal part. The external part contains the System Controller and its receiver. The internal part contains the processing chip and microelectrodes. The communication between each part was via wireless communication chip module. The signal from the uninjured side was collected by preamplifier and AD transfer, then analyzed by the processing chip. Then, stimulating signal was sent out to stimulate the injured side through the V-I transfer. AD, analog-to-digital; V-I, voltage-current.

of the right auricle cartilage and bone border was identified. Skin on the masseter muscle surface and subcutaneous tissues was cut to expose the facial nerve branches. A facial nerve monitoring probe (NTS-2000; NCC Medical Co., Ltd., Shanghai, China) was used to stimulate the nerve branch and masseter muscle and jaw twitches were observed. These nerve branches extend towards the proximal end and join the posterior and superior nerves to form the stylomastoid foramen. When the probe stimulated the stylomastoid foramen, twitches in the neck, cheek, eyelid and ear muscle were detected. A partial facial nerve stem segment $(2-3 \mathrm{~mm})$ at the stylomastoid 


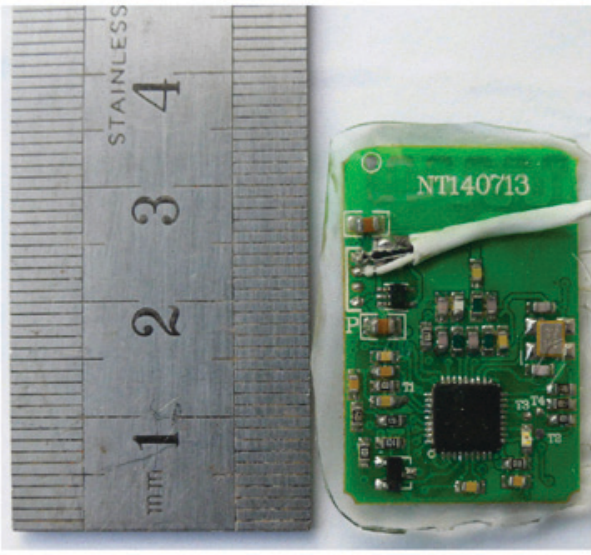

Figure 3. Artificial facial nerve system processing chip. The chip is $3 \times 2 \mathrm{~cm}$, which consists of three parts: The signal collection module, the stimulating module and the wireless communication module. The communication module contains a CC1110 low power consumption MPU chip, with $433 \mathrm{MHz}$ frequency-shift keying communication unit connecting with the MPU through the Serial Peripheral Interface. MPU, microprocessing unit.

foramen was excised and in order to prevent recanalization, the distal and proximal ends were rotated and the severed distal and proximal ends of the nerve were stimulated by the facial nerve monitoring probe $10 \mathrm{~min}$ later. When no twitching was observed in the neck, ears and eyes, disconnection was confirmed. All incisions were sutured and stitches were removed after 7 days to allow for facial expression observation in rabbits. Electromyography (EMG; NTS-2000) was performed to examine orbicularis oculi muscle activity on the surgical (right) side to confirm denervation.

Implantation of the IAFNS. One week after the facial paralysis model was established, all 12 rabbits were subsequently anesthetized with $3 \mathrm{ml} / \mathrm{kg} 1 \%$ pentobarbital sodium via the marginal ear vein. The skin was incised on the parietal transverse surface to expose the skull periosteum and the subcutaneous tissue was separated to make a $4 \times 5 \mathrm{~cm}$ space for insertion of the processing chip. Once the chip was inserted, the subcutaneous tissue was sutured.

The signal collection module of the chip was composed of three electrodes, including two collection electrodes and one reference electrode. Two collection electrodes were embedded into the superior and inferior orbicularis oculi respectively. The reference electrode was embedded into the outer canthus. At implantation the upper and lower eyelid skin was sterilized. A 2-ml guided needle was used to penetrate from the lateral limbus of the eye epidermis to the inside, near the inner canthus. The electrode wire was inserted and positioned in the orbicularis oculi muscle via the guide needle, which was subsequently carefully removed. The reference electrode was embedded into the lateral canthus skin using the same method.

The stimulation module included three-channel stimulation and one reference electrode. Two stimulating electrodes were embedded into the superior orbicularis oculi and the other into the inferior orbicularis oculi. The reference electrode was embedded into the outer canthus as indicated in Fig. 1.

In vivo and in vitro validity and stability of the IAFNS. The IAFNS was run for $5 \mathrm{~h}$ daily for 30 consecutive days.
Table I. Electromagnetic radiation energy level (Horizontal level) under three states.

\begin{tabular}{lrrr}
\hline & \multicolumn{3}{c}{ Electromagnetic } \\
radiation energy level $(\mathrm{dB} \mu \mathrm{V} / \mathrm{M})$ \\
\cline { 2 - 4 } Horizon \\
\cline { 2 - 4 } level $(\mathrm{m})$ & \multicolumn{1}{c}{ State 1} & \multicolumn{1}{c}{ State 2 } & \multicolumn{1}{c}{ State 3 } \\
\hline 0 & $63.97 \pm 0.06$ & $63.97 \pm 3.98$ & $58.7 \pm 1.82$ \\
1 & $64.3 \pm 0.17$ & $62.73 \pm 2.00$ & $55.47 \pm 6.62$ \\
3 & $64.13 \pm 0.12$ & $51.97 \pm 2.06$ & $48.07 \pm 0.81$ \\
10 & $52.07 \pm 1.95$ & $44.97 \pm 0.21$ & $46.3 \pm 2.17$ \\
\hline
\end{tabular}

Data are presented as the mean \pm standard deviation. State 1 , in vitro environment condition; State 2, implanted within the rabbit with the skin not sutured; State 3, implanted within the rabbit with sutured skin.

Table II. Electromagnetic radiation energy level (vertical level) under three states.

\begin{tabular}{lrrr}
\hline & \multicolumn{3}{c}{$\begin{array}{c}\text { Electromagnetic } \\
\text { radiation energy level }(\mathrm{dB} \mu \mathrm{V} / \mathrm{M})\end{array}$} \\
\cline { 2 - 4 } $\begin{array}{l}\text { Vertical } \\
\text { level }(\mathrm{m})\end{array}$ & \multicolumn{1}{c}{ State 1 } & \multicolumn{1}{c}{ State 2 } & \multicolumn{1}{c}{ State 3 } \\
\hline 0 & $57.5 \pm 0.00$ & $57.37 \pm 3.50$ & $51.4 \pm 1.47$ \\
1 & $63.93 \pm 0.06$ & $56.2 \pm 4.94$ & $56.4 \pm 0.20$ \\
3 & $63.47 \pm 0.49$ & $51.77 \pm 1.40$ & $51.67 \pm 1.10$ \\
10 & $41.4 \pm 1.57$ & $40.87 \pm 0.75$ & $42.17 \pm 2.35$
\end{tabular}

Data are presented as the mean \pm standard deviation. State 1 , in vitro environment condition; State 2, implanted within the rabbit with the skin not sutured; State 3, implanted within the rabbit with sutured skin.

Communication between the processing chip and the System Controller was established every 3 days to control and observe the working state of the processing chip. The stimulation frequencies were 30 and $50 \mathrm{~Hz}$ (7). Every 3 days, the smallest stimulus intensity that could induce the movement of the paralyzed muscle was recorded. At $0,10,20$ and 30 days after the surgery, the uninjured cornea was stimulated 20 times, using a cotton bud to irritate the uninjured eyelid to close. The eyelid movement of the injured side was recorded at the same time to evaluate the synchronization of both sides (8).

Statistical analysis. SAS 9.4 (SAS Institute, Cary, NC, USA) was used for statistical processing (t-tests). Data are presented as the mean \pm standard deviation. $\mathrm{P}<0.05$ was considered to indicate a statistically significant difference.

\section{Results}

Experimental animals. A total of 12 male rabbits were evaluated. One rabbit (no. 11) was sacrificed by anesthetic injection via the marginal ear vein $(150 \mathrm{mg} / \mathrm{kg}, 1 \%$ pentobarbital 

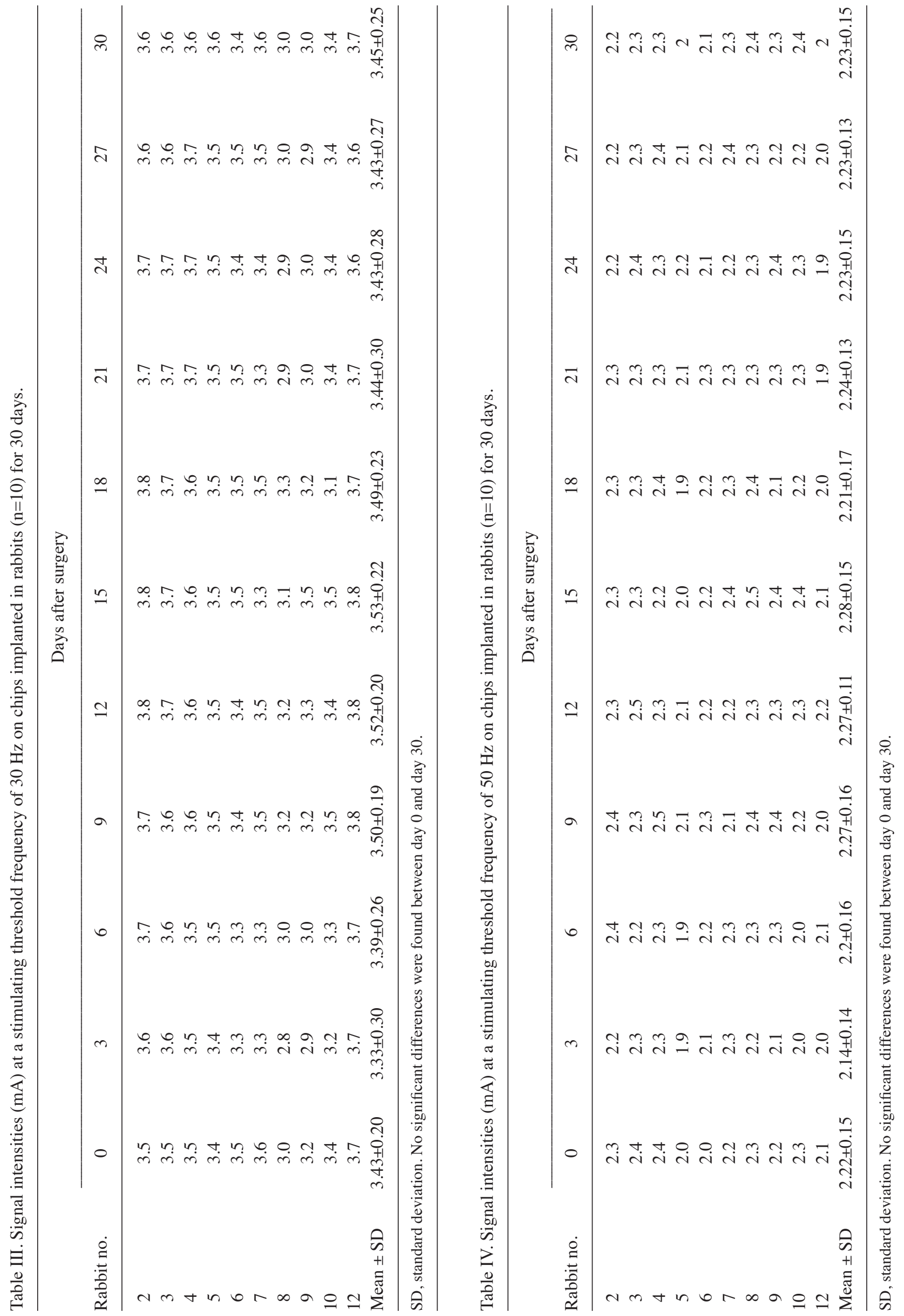
sodium) as no signal was received from the chip 25 days after implantation. Another rabbit (no. 1) succumbed to mortality 4 days following facial paralysis surgery as a result of infection. The 10 remaining rabbits were sacrificed by anesthetic injection $(150 \mathrm{mg} / \mathrm{kg}, 1 \%$ pentobarbital sodium) via the marginal ear vein after research.

Anti-interference test and electromagnetism yield in radiation assessment of the processing chip. Chips ran stably according to the anti-interference test, and signal emission performance did not decrease following the test, indicating results of level A. The results of electromagnetism yield in radiation test (Tables I and II) indicated that the processing chip implanted in rabbits with the skin not sutured had the highest radiation field intensity, compared with the in vitro state and implanted state in sutured rabbits. The lowest intensity of the three states was exhibited when the chip was implanted and sutured in the skin. In each state, radiation field intensity decreased with increasing distance and the minimum intensity observed was at $10 \mathrm{~m}$.

Implantation of the IAFNS. All rabbits were healthy and underwent facial neurosurgery to induce facial paralysis on the right side of the face. During surgery, the denervated distal stump was stimulated with a nerve monitoring system probe. Tics in the ocular region and mouth corner were accompanied by an electromyography monitoring sound, which appeared at the same time the facial nerve was transected. After $10 \mathrm{~min}$, the denervated proximal stump was stimulated with the nerve monitoring system probe, no tics and monitoring sound were detected in the left external ear, ocular region, mouth corner or cervical muscle. One day after the surgery, rabbits exhibited an inability to close the right eyelid and the mouth corner was deviated. Seven days after the surgery, EMG was performed, which revealed denervated muscle on the injured side in all experimental rabbits (sensitivity, $100 \mu \mathrm{V} / \mathrm{D}$; scanning speed, $50 \mathrm{msec} / \mathrm{D}$ ), indicating successful establishment of unilateral peripheral facial paralysis. The processing chips were implanted into the animal model and communication with the System Controller was established.

In vivo and in vitro validity and stability of the IAFNS. The stimulating threshold, which was the minimum stimulus intensity that induced closure of the eye on the paralyzed side, was defined as $50 \mu \mathrm{V}$, according to a previous study (9). Throughout the 30-days experimental period, the threshold of each rabbit was measured every 3 days at two different frequencies (30 and $50 \mathrm{~Hz}$ ). Positive and negative square waves were used at a current intensity of 1-6 mA. The stimulation frequencies used were 30 and $50 \mathrm{~Hz}$ and the mean intensity was $3.45 \pm 0.24 \mathrm{~mA}$ and $2.23 \pm 0.14 \mathrm{~mA}$, respectively. Results demonstrated no significant difference in the current intensity threshold of the stimulating electrode between 0 and 30 days post-surgery in rabbits with the skin sutured (Tables III and IV).

At 0, 10, 20 and 30 days after the surgery, the uninjured cornea was stimulated 20 times in facial nerve paralyzed rabbits using a cotton bud to irritate the uninjured eyelid to close. The electrical stimulator, triggered by the computer, emitted the corresponding electrical stimulation to the injured orbicularis oculi muscle. The number of contractions from the
Table V. Number of times orbicularis oculi muscle contraction was exhibited when the uninjured eyes of rabbits $(n=10)$ were stimulated to close 20 times.

Days after surgery

\begin{tabular}{ccccc}
\cline { 2 - 5 } Rabbit no. & 0 & 10 & 20 & 30 \\
\hline 2 & 20 & 20 & 19 & 19 \\
3 & 20 & 19 & 19 & 20 \\
4 & 20 & 20 & 19 & 20 \\
5 & 19 & 19 & 20 & 20 \\
6 & 20 & 20 & 19 & 19 \\
7 & 19 & 20 & 20 & 19 \\
8 & 20 & 19 & 20 & 19 \\
9 & 20 & 19 & 20 & 20 \\
10 & 20 & 20 & 20 & 19 \\
12 & 18 & 19 & 19 & 18 \\
\hline
\end{tabular}

injured orbicularis oculi muscle triggered by the uninjured side is indicated in Table V. Almost all cases of stimulation induced muscle movement in the injured side. No significant differences were found between the two groups at any time point.

\section{Discussion}

At present, all treatments for facial nerve decompression are directed to the facial nerve, with the primary purpose of restoring its anatomical and functional integrity (10). However, the structure and function of the facial nerve in some patients does not fully recover (11-13). Sönmez et al (14) implanted a weight in the upper eyelid to close the eyelid with gravity and prevent blindness complications, but this results in poor quality of life.

A previous study focused on restoration of the paralyzed muscle instead of nerve function (15). Muscle movement information was extracted by multi-channel electroencephalogram large matrix. An electronic processing chip was able to process the movement information and control the electrical stimulator to release current, which directly induced skeletal muscle movement. However, the signal was weak and the data flow processing was time consuming. Therefore, this method may be applicable to slow action movements alone and may not be suitable for rapid movements, such as the facial muscles.

With appropriate currents directly stimulating the muscle, safety and effectiveness, the use of implantable electronic devices has been accepted by the majority of scholars (16). Implantable electronic devices have been widely applied in the biomedical field, including cochlear implants and artificial prosthesis control system devices $(15,17)$. The movement of a prosthesis may be controlled by gathering and analyzing muscle electromyography signals (18).

The IAFNS demonstrated in the present study may be used clinically in three ways. Firstly, the artificial nerve system may be used to replace the facial nerve center and facial nerve, to stimulate the facial muscles directly, to restore expression and 
maintain face symmetry. This may be used to treat patients with facial paralysis and restore facial muscle contraction function. Secondly, the artificial nerve system may be used to temporarily maintain the facial muscle tension for a patient being prepared for facial nerve transplantation in order to prevent facial muscle disuse atrophy and may be stopped once facial nerve function has recovered. Thirdly, the artificial nerve system may be used as a supplement for facial nerve transplantation in the instance that neural transplantation is invalid or is ineffective. In this case, the system may be able to stimulate facial muscles to provide the extra contraction strength, rendering symmetrical movement in the paralyzed and contralateral areas.

In a previous study by the current authors (9), the time domain and peak absolute potentials of EMG signals during the natural continuous eye-opening, natural continuous eye-closing, natural blinking and evoked eye-closing states were compared. Absolute potentials of initial myoelectric signals, which triggered the orbicularis oculi muscle to potently contract during natural blinking and evoked eye-closing states, were $55.39 \pm 6.25$ and $52.16 \pm 3.69 \mu \mathrm{V}$, respectively; therefore, the chip stimulating threshold was determined as $50 \mu \mathrm{V}$.

The positions of the stimulating electrode were determined according to previous research (19). The optimal position is able to improve the utilization efficiency of the stimulus current and therefore the minimum current may be used to achieve the largest muscles contraction.

In the present study, stimulation frequencies were determined predominantly by the eyelid movement stimulated in the facial nerve paralyzed rabbits. At first, stimulation frequencies of 20, 22, 25, 30, $50 \mathrm{~Hz}$ were assessed: 20, 22 and $25 \mathrm{~Hz}$ could induce orbicularis oculi muscle movement, but these movements were too weak to close the eyelids completely. At frequencies of 30 and $50 \mathrm{~Hz}$, the paralyzed orbicularis oculi muscle could be stimulated to provoke the complete closure of eyes in the facial nerve paralyzed rabbits, with 30 and $50 \mathrm{~Hz}$ yielding mean intensities of 3.45 and $2.23 \mathrm{~mA}$, respectively. There were no significant differences in the stimulation intensities between 0 and 30 days after implantation. The results indicated that the artificial facial nerve system reconstructed the nerve reflex arc that was damaged by induced facial nerve injury.

In the present study, 30 days after implantation in the animal model, the processing chip worked stably. The synchronicity of the movement on both sides was assessed on 0, 10, 20 and 30 days after implantation. Results indicated that high synchronicity was obtained and length of time the implant was embedded within the rabbits did not affect the results.

At the Shanghai Testing \& Inspection Institute for Electrical Equipment, the anti-interference ability of the implantable artificial facial nerve system chip was assessed and results confirmed that the system was not affected by high intensity radiation during operation. In addition, the present study also evaluated the radiation field intensity of the artificial facial nerve chip under states 1,2 and 3. Test distances analyzed were $0,1,3$ and $10 \mathrm{~m}$. Results indicated that most of the radiation field intensity was $<60 \mathrm{~dB}$ at three states, except $0 \mathrm{~m}$ in state 3 . In further research, the processing chip will be coated with insulating material; the chip was in a bare-metal state in this research, which led to a high radiation field intensity.

In conclusion, the present study indicated that the artificial facial nerve system applied an appropriate muscle stimulation signals to produce the corresponding muscle contractions to perform specific actions. Implantation of the artificial facial nerve system into rabbits restored the function of induced facial paralysis on the paralyzed side and provoked eye closing and blinking. Furthermore, the system functioned well while implanted in the body. From the findings of the present study, it is suggested that the wireless communication between the processing chip and the system control program is reliable, which could displayed the contralateral electromyography activity and the working state of the implanted chip, adjust the appropriate parameters to reconstruct the paralyzed muscle.

\section{Acknowledgements}

The authors are thankful for financial support received from the Science and Technology Commission of Shanghai Key Research Projects (grant no. 11441900102).

\section{References}

1. Lu Xg, Cai Zg, Yu Gy and Peng X: Surgical treatment of transected peripheral facial nerve injury. Beijing Da Xue Xue Bao 43: 106-111, 2011 (In Chinese).

2. Sardesai MG and Moe K: Recent progress in facial paralysis: Advances and obstacles. Curr Opin Otolaryngol Head Neck Surg 18: 266-271, 2010.

3. Volk GF, Pantel M and Guntinas-Lichius O: Orlando Guntinas-Lichius: Concepts in facial nerve reconstruction. Head Face Med 6: 25, 2010.

4. Langhals NB, Urbanchek MG, Ray A and Brenner MJ: Update in facial nerve paralysis: Tissue engineering and new technologies. Curr Opin Otolaryngol Head Neck Surg 22: 291-299, 2014.

5. Wang YJ, Li KY, Liu JQ, Xu DY, Rui YF and Yan CS: Artificial facial nerve reflex restores eyelid closure following orbicularis oculi muscle denervation. Neural Regen Res 5: 1750-1755, 2010.

6. Zhang Y, Li KY, Jin C, Wang YT, Geng L, Sun YJ, Tian HC, Liu JQ and Jin XJ: Comparative studies on the implantation of nanoplatinum black and pure platinum electrodes in the rabbit orbicularis oculi muscle. J Laryngol Otol 128, 679-689, 2014

7. Jie T, Zhiqiang G, Guodong F, Yubin X, Xiuyong D, Tingting C and Yang Z: The effective stimulating pulse for restoration of blink function in unilateral facial nerve paralysis rabbits, verified by a simple FES system. Eur Arch Otorhinolaryngol 273: 2959-2964, 2016

8. Yi X, Jia J, Deng S, Shen SG, Xie Q and Wang G: A blink restoration system with contralateral EMG triggered stimulation and real-time artifact blanking. IEEE Trans Biomed Circuits Syst 7: 140-148, 2013.

9. Dongyue X, Keyong L, Jingquan L, Yujuan W and Yuefeng R: Quantitative features of myoelectric signals in the orbicularis oculi muscle during different motion states. Neural Regen Res 5: 1895-9, 2010.

10. Hadlock T: Facial paralysis: Research and future directions. Facial Plast Surg 24: 260-267, 2008.

11. Shi Y, Zhou L, Tian J and Wang Y: Transplantation of neural stem cells overexpressing glia-derived neurotrophic factor promotes facial nerve regeneration. Acta Otolaryngol 129: 906-914, 2009.

12. Bozkurt G, Mothe AJ, Zahir T, Kim H, Shoichet MS and Tator $\mathrm{CH}$ : Chitosan channels containing spinal cord-derived stem/progenitor cells for repair of subacute spinal cord injury in the rat. Neurosurgery 67: 1733-1744, 2010.

13. Kabatas S and Teng YD: Potential roles of the neural stem cell in the restoration of the injured spinal cord: Review of the literature. Turk Neurosurg 20: 103-110, 2010.

14. Sönmez A, Oztürk N, Durmuş N, Bayramiçli M and Numanoğlu A: Patients' perspectives on the ocular symptoms of facial paralysis after gold weight implantation. J Plast Reconstr Aesthet Surg 61: 1065-1068, 2008. 
15. Pohlmeyer EA, Oby ER, Perreault EJ, Solla SA, Kilgore KL, Kirsch RF and Miller LE: Toward the restoration of hand use to a paralyzed monkey: Brain-controlled functional electrical stimulation of forearm muscles. PLoS One 4: e5924, 2009.

16. Laizou PC: Signal-processing techniques for cochlear implants. IEEE Eng Med Biol Mag 18: 34-46, 1999.

17. Hochberg LR, Serruya MD, Friehs GM, Mukand JA, Saleh M, Caplan AH, Branner A, Chen D, Penn RD and Donoghue JP: Neuronal ensemble control of prosthetic devices by a human with tetraplegia. Nature 442: 164-171, 2006.
18. Wessberg J, Stambaugh CR, Kralik JD, Beck PD, Laubach M, Chapin JK, Kim J, Biggs SJ, Srinivasan MA and Nicolelis MA: Real-time prediction of hand trajectory by ensembles of cortical neurons in primates. Nature 408: 361-365, 2000.

19. Zhang Y, Li K, Jin C, Wang Y, Geng L, Sun YJ and Tian H: Electrical stimulation characteristics of denervated orbicularis oculi muscle. Neurol Sci 36: 1379-1386, 2015. 